\title{
Prognostic significance of contactin 3 expression and associated genes in glioblastoma multiforme
}

\author{
YI-FANG ZHU ${ }^{1,2}$, YUAN-BIAO GUO ${ }^{2}$, HAN-YU ZHANG ${ }^{3}$, PENG YANG $^{4}$, \\ DAN-FENG WEI $^{2}$, TONG-TONG ZHANG ${ }^{2}$, BI-RAN PAN ${ }^{2}$ and LEI LIU ${ }^{2}$ \\ ${ }^{1}$ Clinical Laboratory, Sichuan Provincial Orthopedic Hospital, Chengdu, Sichuan 610041; \\ ${ }^{2}$ Medical Research Center; ${ }^{3}$ Department of Neurology; ${ }^{4}$ Pathology Laboratory, The Second Affiliated Clinical \\ College of Chongqing Medical University, The Third People's Hospital of Chengdu, Chengdu, Sichuan 610031, P.R. China
}

Received September 6, 2018; Accepted May 13, 2019

DOI: $10.3892 / 01.2019 .10482$

\begin{abstract}
Contactin 3 (CNTN3) is a member of the contactin family that is primarily expressed in the nervous system. However, to the best of our knowledge, expression of contactin and its role in the development and progression of brain tumours has not been studied. Although glioblastoma multiforme (GBM) is the most common malignant brain tumour, advances in therapeutic options for patients with GBM have been modest due to an incomplete understanding of the molecular mechanisms underlying development and progression. The aim of the present study was to examine the correlation between CNTN3 and its associated genes and the clinical outcome in patients with GBM. CNTN3 and the expression levels of associated genes were analysed in GBM datasets obtained from the SAGE Anatomical viewer website, Gene Expression Omnibus, Oncomine and The Cancer Genome Atlas. CNTN3 was significantly downregulated in patients with GBM. Subsequently, the expression of CNTN3 was further validated using immunohistochemistry in a cohort of GBM specimens. The immunohistochemistry results were consistent with the in silico analyses. Kaplan-Meier analysis indicated that patients with lower expression levels of CNTN3 had a significantly shorter overall survival (OS) time compared with patients with higher levels of CNTN3 expression. Univariate and multivariate Cox regression analyses demonstrated that CNTN3 expression was an independent prognostic indicator in patients with GBM. Furthermore, gene set enrichment analysis revealed that $C N T N 3$ was associated with the receptor tyrosine-protein kinase (ErbB) signalling pathway. In the ErbB signalling pathway, epidermal growth factor receptor (EGFR) was negatively correlated with CNTN3. Taken together, these
\end{abstract}

Correspondence to: Mrs. Yi-Fang Zhu, Clinical Laboratory, Sichuan Provincial Orthopedic Hospital, 132 West Section 1, Yihuan Road, Wuhou, Chengdu, Sichuan 610041, P.R. China

E-mail: 53113072@qq.com

Key words: glioblastoma multiforme, contactin 3, prognosis, in silico analysis, receptor tyrosine-protein kinase pathway, epidermal growth factor receptor data suggest that lower expression levels of CNTN3 may be an independent biomarker that predicts poor OS time in patients with GBM, and that EGFR expression in the ErbB pathway may be associated with CNTN3 expression.

\section{Introduction}

Glioblastoma, also known as glioblastoma multiforme (GBM), is the most common cancer of the central nervous system, accounting for $>80 \%$ of all brain tumours (1). GBM has an annual incidence of 5.26 cases per 100,000 individuals, with 17,000 new cases being diagnosed each year (2). Due to rapid proliferative rate and a characteristic invasive nature, the survival rate of patients with GBM remains low (1). Typically, patients with GBM survive for 12-15 months after diagnosis, with only $3-5 \%$ of individuals surviving $>5$ years (3). The underlying cause of the majority of cases is unclear. Identifying biomarkers of GBM may improve the diagnosis and prognosis of patients, and provide novel therapeutic targets for treating patients with GBM.

The contactin (CNTN) proteins are a subgroup of proteins, which belong to the immunoglobulin (Ig) superfamily of proteins, and are primarily expressed in the nervous system. CNTNs consist of six members: CNTN1, CNTN2 [transient axonal glycoprotein 1 (TAG-1)], CNTN3 [brefeldin A-inhibited guanine nucleotide-exchange protein 1 (BIG)-1; plasmacytoma-associated neuronal glycoprotein (PANG)], CNTN4 (BIG-2), CNTN5 (neural recognition molecule NB-2) and CNTN6 (neural recognition molecule NB-3) (4). The expression of CNTN3 mRNA is developmentally regulated and reaches its highest level in the adult brain (5). CNTN3 is also termed PANG or BIG-1, and was discovered in the endoplasmic reticulum of plasmacytomas (6). CNTN3 is a membrane protein anchored by glycosylphosphatidylinositol, with six Ig-like domains and four-fibronectin type III repeats, and it belongs to the TAG-1/F3 subgroup of the Ig superfamily of proteins (5). The CNTN3 gene is located at 3 p26 in the genome (7) and its expression is restricted to certain subsets of neurons, including the cerebellar Purkinje cells, the granule cells of the dentate gyrus and the neurons in the superficial layers of the cerebral cortex (1). CNTN3 may function in the formation and maintenance of specific neuronal networks (6,8-11). Bouyain and Watkins (12) found 
that CNTN3 interacts with the receptor protein tyrosine phosphatases $\gamma$ and is involved in the construction of neural networks. The expression and function of CNTN3 in different types of cancer have not been thoroughly investigated. A previous study suggested that CNTN3 is a potential target gene of hsa-miR-3675b in breast cancer, and by using gene ontology analysis, it was demonstrated that CNTN3 may be associated with cell proliferation, apoptosis and cell cycle progression (13). However, to the best of our knowledge, the biological role of CNTN3 in GBM remains unknown.

In the present study, microarrays and sequencing datasets were analysed to elucidate the clinical value of CNTN3 in GBM and the associated molecular mechanisms. The expression of CNTN3 in GBM was compared with that in normal tissues using SAGE Anatomical viewer, Gene Expression Omnibus (GEO), Oncomine and The Cancer Genome Atlas (TCGA) databases. The expression of the CNTN3 protein was examined in microarrays from GBM tissues. The clinical data were used to determine the prognostic value of CNTN3 in patients with GBM. The pathways associated with CNTN3 were determined using gene set enrichment analysis (GSEA) and interaction networks were constructed using Search Tool for the Retrieval of Interacting Genes/Proteins (STRING) database and Cytoscape analysis.

\section{Materials and methods}

SAGE anatomical viewer. The SAGE Anatomical Viewer (cgap.nci.nih.gov/sage/anatomicviewer) is a website established by the National Cancer Institute (Bethesda, MD, USA) that displays gene expression in normal and malignant human tissues by shading a given organ in one of 10 colours, depicting differing levels of gene expression. CNTN3 expression (the NM_020872 transcript) was found to be present in both normal and cancer tissues. The tag with the highest rank and the highest frequency was selected for CNTN3 gene, and the sequence was AGATATGCAC.

GEO and TCGA datasets. GEO is a public functional genomics data repository in which gene array and sequence-based data are accepted (https://www.ncbi.nlm.nih.gov/geo/). GSE4290 (14) and GSE7696 (15) microarray datasets were obtained from the GEO database. GSE4290 contained data from 81 GBM samples and 23 samples from patients with epilepsy, which were used as the non-tumour samples. GSE7696 contained samples from 80 patients with GBM whom participated in clinical trials and 4 samples of normal brain tissue. Transcription and clinical data for patients with GBM were also downloaded from the TCGA website (https://cancergenome.nih.gov/), and consisted of 156 GBM samples and 5 normal tissues.

Data processing. Raw data were downloaded and normalised using the multi-array average analysis method (16) in $\mathrm{R}$ version 3.4.4 (17). Differentially expressed genes were identified with the Limma package (18), and a fold change of $>2$ and $\mathrm{P}<0.05$ were set as the cut-offs.

Oncomine database analysis. CNTN3 mRNA levels in normal and GBM tissues were compared in the Oncomine database (www.oncomine.org). CNTN3 mRNA levels were downregulated in the GBM samples from the 'Sun Brain' (14) and 'Murat Brain' (15) datasets in the Oncomine database. CNTN3 levels were also compared across these two datasets. The rank for a gene was the median rank for that gene across each of the analyses. The P-value for a gene was its P-value for the median-ranked analysis.

Immunohistochemistry.A GBM tissue microarray was purchased from Shanghai Outdo Biotech Co., Ltd., which contained 67 GBM and 13 adjacent normal brain tissue samples. All of the patients had been pathologically diagnosed with GBM. The thickness of tissue sections was $4 \mu \mathrm{m}$. Two serial tissue sections were prepared for CNTN3 and EGFR detection. The tissue slides were deparaffinised in xylene and rehydrated gradually in 100,85 and $75 \%$ alcohol. Next, the slides were treated with $3 \%$ $\mathrm{H}_{2} \mathrm{O}_{2}$ for $15 \mathrm{~min}$, autoclaved in $10 \mathrm{mM}$ citric sodium ( $\mathrm{pH}$ 6.0) for $30 \mathrm{~min}$ to permeabilize the samples, rinsed in PBS and then incubated with a primary antibody against CNTN3 (Abcam; cat. no. ab203592; 1:100) or EGFR (Proteintech; cat. no. 18986-1-AP; $1: 500)$ at $4^{\circ} \mathrm{C}$ overnight. This was followed by incubation with biotinylated anti-rabbit IgG (OriGene Technologies, Inc.; cat. no. TA130016; 1:200) for $30 \mathrm{~min}$ at $37^{\circ} \mathrm{C}$ and then peroxidase-labelled streptavidin (OriGene Technologies, Inc.; cat. no. AR100017; 1:100) for $15 \mathrm{~min}$ at $37^{\circ} \mathrm{C}$. The signals were visualised using a diaminobenzidine substrate kit (ZSGB-BIO; OriGene Technologies, Inc.) according to the manufacturer's protocol. CNTN3 immunostaining score was determined using a semi-quantitative approach. The percentage of stained cells in each sample was scored as follows: 0,$0 ; 1,1-25 ; 2,26-50$; $3,51-75$; and $4,76-100 \%$. The intensity of staining was scored as follows: 0 , negative; 1 , weak staining; 2 , moderate staining; and 3 , strong staining. The final staining score was the intensity score multiplied by the percentage score. A final staining score $<6$ was classified as negative CNTN3/EGFR expression and a score $\geq 6$ was classified as positive CNTN3/EGFR expression.

Statistical analysis. The mRNA expression and protein expression between different groups was compared respectively using t-test and Mann Whitney U test, and correlations were evaluated using Pearson's correlation analyses. The associations between the CNTN3 expression levels and the clinicopathological features were evaluated using $\chi^{2}$ test. Kaplan-Meier estimator curves and log-rank test were used for the survival analysis. Univariate and multivariate survival analyses were performed using Cox's proportional hazards model. The data are presented as the mean \pm the standard error the mean. $\mathrm{P}<0.05$ was considered to indicate a statistically significant difference. All of the statistical analyses were performed using SPSS version 21.0 (IBM Corp.).

Identifying potential molecular mechanisms of CNTN3 expression in GBM. GSEA 3.0 (Broad Institute, Inc.) (19) was used to detect the expression changes of gene sets associated with CNTN3. The annotated gene sets $\mathrm{c} 2 . \mathrm{cp}$. kegg.v6.0.symbols.gmt were selected as the reference gene sets. A false-discovery-rate of $<0.05$ was used as the cut-off criteria. STRING (http://string-db.org) was used to identify the potential interaction networks between proteins encoded by these genes. Cytoscape version 2.8.3 (20) software was used to construct interaction networks between the proteins in GBM. 
A

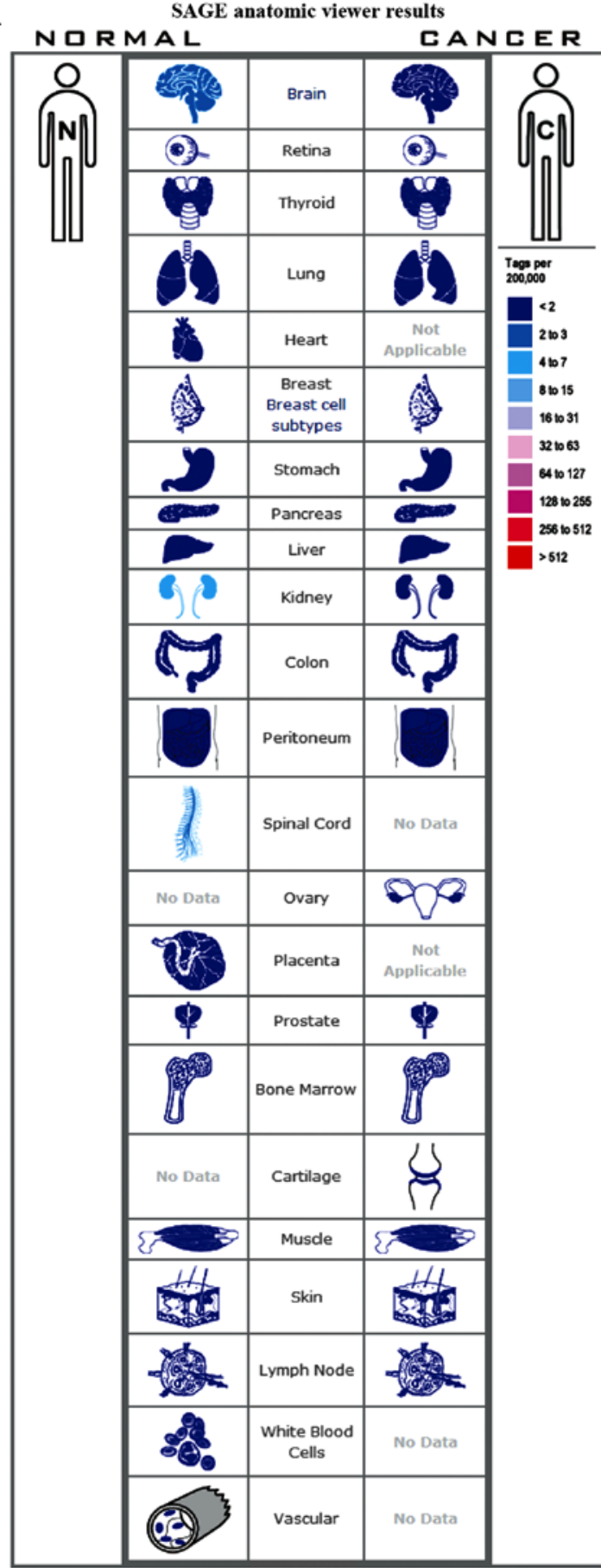

SAGE anatomic viewer results
B
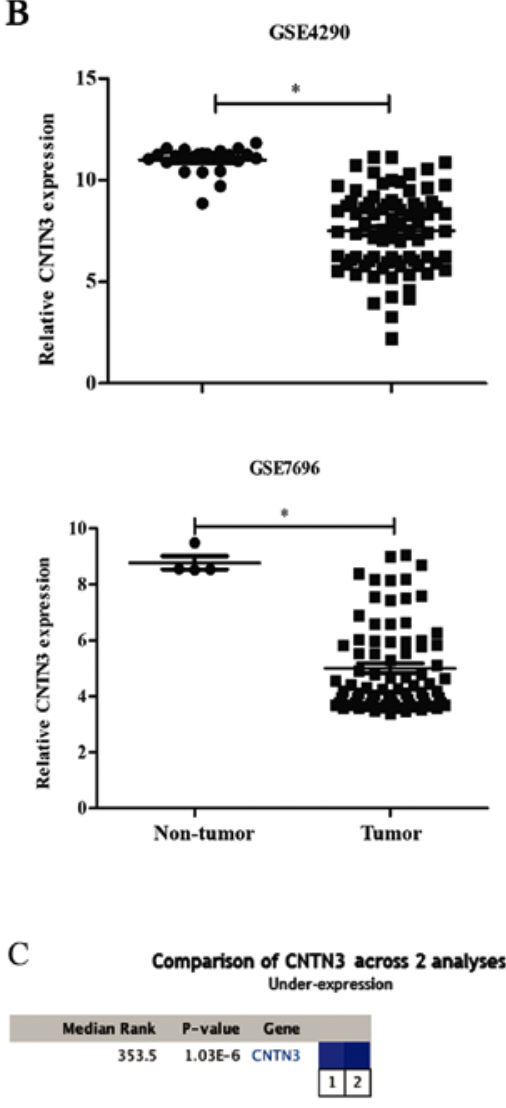

Legend

1. Globlastoma vs. Normal

Murat Brain, J Clin Oncol, 2008

2. GEoblastoma vs. Normal

Sun Brain, Cancer Cell, 2006

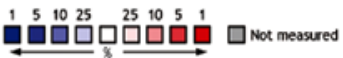

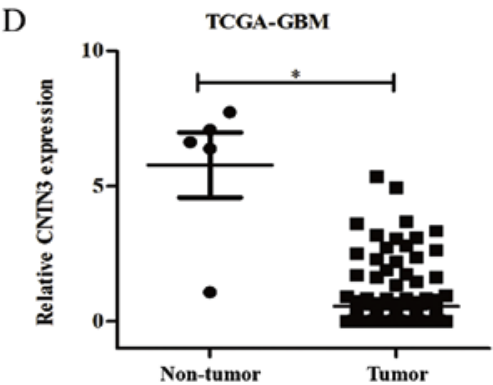

Figure 1. CNTN3 expression is downregulated in patients with GBM. (A) SAGE anatomic viewer expression profile for CNTN3 was compared in a range of different types of cancer and normal tissues. (B) Analysis of CNTN3 expression levels in patients with GBM and normal tissues from two gene datasets. " $\mathrm{P}<0.0001$. (C) A meta-analysis of $C N T N 3$ gene expression from two Oncomine databases where the coloured squares indicate the median rank of CNTN3. (D) The Cancer Genome Atlas dataset was used for bioinformatics analysis to validate CNTN3 mRNA expression in GBM. CNTN3, contactin 3; GBM, glioblastoma multiforme ${ }^{*} \mathrm{P}<0.0001$.

\section{Results}

CNTN3 gene expression in GBM and normal tissues. The gene expression levels of $C N T N 3$ between cancer and matched normal tissues were analysed using SAGE anatomical viewer with tag. CNTN3 mRNA expression was downregulated in brain and kidney cancer tissues when compared with that in normal tissues (Fig. 1A). Analysing the GEO datasets revealed that the gene expression level of $C N T N 3$ was significantly lower in GBM compared with that in normal tissue in the two datasets (both $\mathrm{P}<0.05$; Fig. 1B). CNTN3 expression was downregulated in the GSE4290 (fold-change, -12.218; $\mathrm{P}=4.64 \times 10^{-26}$ ) and GSE7696 (fold-change, $-13.6 ; \mathrm{P}=2.07 \times 10^{-06}$ ) datasets. The median rank of CNTN3 was 353.3 based on a meta-analysis across the two datasets using Oncomine algorithms ( $\mathrm{P}=1.03 \times 10^{-6}$; Fig. $\left.1 \mathrm{C}\right)$. Similarly, CNTN3 was also downregulated in GBM in the dataset obtained from TCGA (fold-change, -5.855; P=1.32×10-24; Fig. 1D). 
A

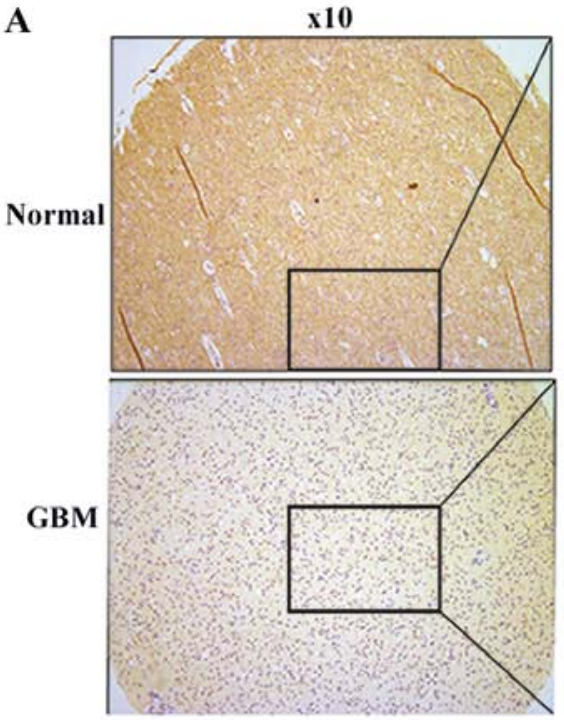

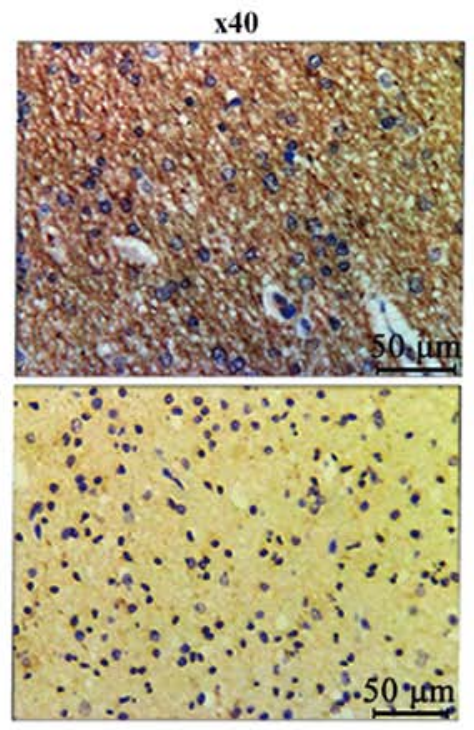

B

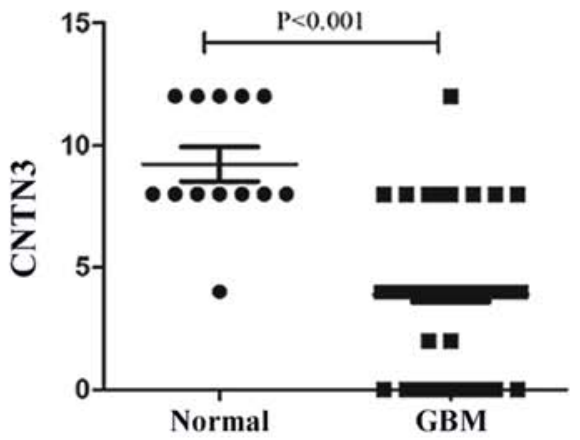

Figure 2. Expression of CNTN3 in GBM and adjacent normal brain tissues. (A) Representative immunohistochemical stains of CNTN3 expression in GBM and normal brain tissues. (B) CNTN3 expression was significantly higher in the normal tissues compared with that in the GBM tissues. The scores of CNTN3 in GBM and normal brain tissues were analysed by Mann Whitney U test. CNTN3, contactin 3; GBM, glioblastoma multiforme.
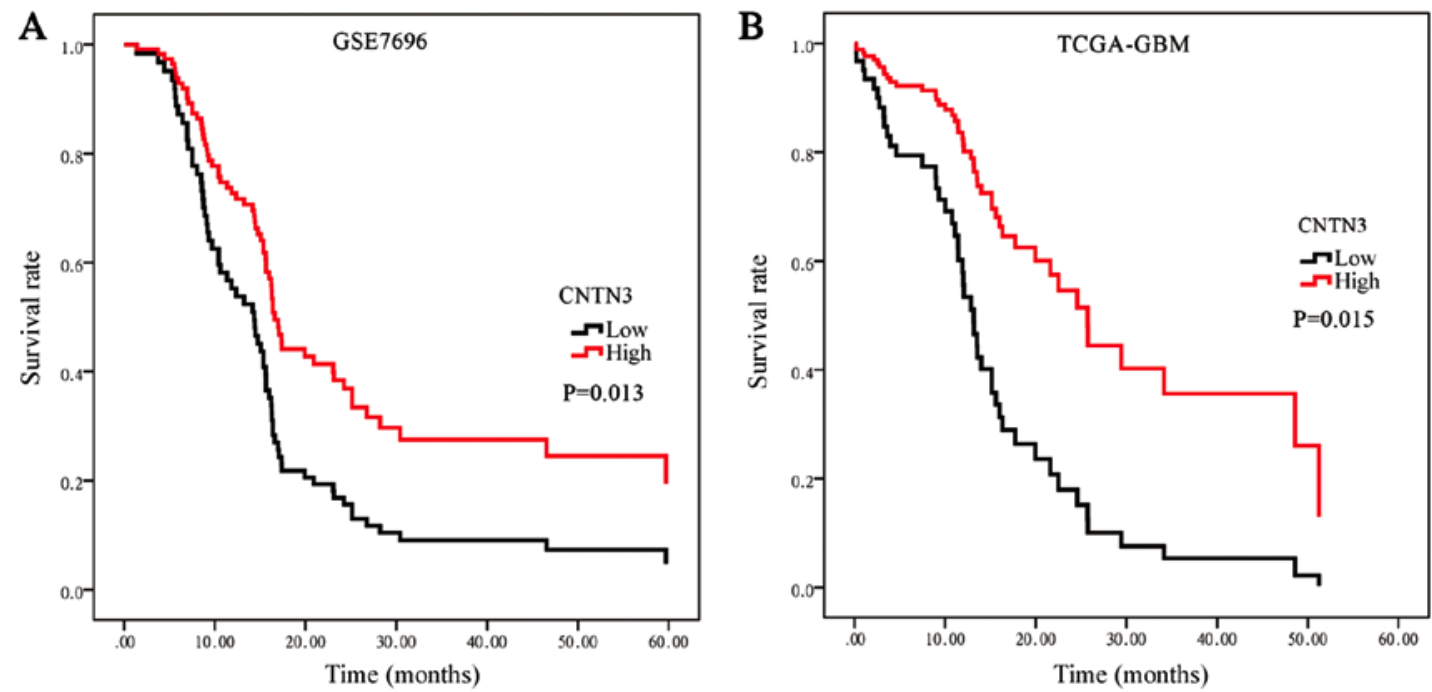

Figure 3. Kaplan-Meier analysis of OS in patients with GBM. (A) OS analysis of patients with GBM based on CNTN3 mRNA expression levels in the GSE7696 dataset. Cutoff value, 4.3; $\mathrm{P}=0.013$. (B) Association between $C N T N 3$ expression and OS was performed on TCGA dataset. Cutoff value, 1.1; $\mathrm{P}=0.015$. CNTN3, contactin 3; GBM, glioblastoma multiforme; OS, overall survival; TCGA, The Cancer Genome Atlas.

Immunohistochemistry staining was performed on the GBM tissue microarray. CNTN3 was primarily located in the cytoplasm of neuronal cells and its expression was downregulated in GBM tissues (Fig. 2A). Positive CNTN3 expression was observed in $92.3 \%(12 / 13)$ of the adjacent normal brain tissues and in $13.4 \%(9 / 67)$ in the GBM tissues ( $\mathrm{P}<0.0001$; Fig. 2B).

Clinical and prognostic value of CNTN3 in GBM patients. The association between CNTN3 expression and the clinicopathological features of patients was statistically analysed in the GSE7696 dataset as it had survival data and other clinical data. Clinical parameters consisted of sex, age, treatment method (temozolomide with radiotherapy or radiotherapy alone) and O6-methylguanine-DNA methyltransferase
(MGMT) methylation status. There was no significant association between CNTN3 expression and any of the parameters (all P>0.05; Table I).

To determine the prognostic significance of CNTN3 expression in patients with GBM, Kaplan-Meier survival analysis was used to evaluate the association between CNTN3 expression and survival in the GS7696 dataset (Fig. 3A). The data was divided into high- and low-expression subgroups based on the median CNTN3 expression, 15.58. The median overall survival (OS) time was 14.140 months [95\% confidence interval (CI), 10.933-17.347] in the low expression group and 17.070 months (95\% CI, 15.288-18.852) in the high expression group. A log-rank test demonstrated that patients with low CNTN3 expression had a significantly shorter OS time compared with those with high $\mathrm{CNTN} 3$ expression $(\mathrm{P}=0.013$; 
Table I. Association of CNTN3 expression with the clinicopathological characteristics of patients with GBM.

\begin{tabular}{|c|c|c|c|c|}
\hline \multirow[b]{2}{*}{ Clinicopathological characteristics } & \multirow[b]{2}{*}{ Total, $\mathrm{n}$} & \multicolumn{2}{|c|}{ CNTN3 expression } & \multirow[b]{2}{*}{ P-value } \\
\hline & & Low, n & High, $\mathrm{n}$ & \\
\hline Sex & & & & 0.799 \\
\hline Male & 59 & 29 & 30 & \\
\hline Female & 21 & 11 & 10 & \\
\hline Age, years & & & & 0.264 \\
\hline$\leq 60$ & 64 & 30 & 34 & \\
\hline$>60$ & 16 & 10 & 6 & \\
\hline Treatment & & & & 0.348 \\
\hline $\mathrm{TMZ}$ + radiotherapy & 52 & 24 & 28 & \\
\hline Radiotherapy & 28 & 16 & 12 & \\
\hline MGMT & & & & 0.171 \\
\hline Methylation & 44 & 19 & 25 & \\
\hline No methylation & 34 & 20 & 14 & \\
\hline Unknown & 2 & 1 & 1 & \\
\hline
\end{tabular}

CNTN3, contactin 3; GBM, glioblastoma multiforme; TMZ, temozolomide; MGMT, O6-methylguanine-DNA methyltransferase.

Table II. Univariate and multivariate Cox proportional hazard regression analysis.

\begin{tabular}{|c|c|c|c|c|c|c|}
\hline \multirow[b]{2}{*}{ Clinicopathological characteristic } & \multicolumn{3}{|c|}{ Univariate analysis } & \multicolumn{3}{|c|}{ Multivariate analysis } \\
\hline & HR & $95 \% \mathrm{CI}$ & P-value & HR & $95 \% \mathrm{CI}$ & P-value \\
\hline Age, $\geq 60$ vs. $<60$ years & 1.033 & $1.003-1.065$ & $0.031^{\mathrm{a}}$ & 1.019 & $0.990-1.048$ & 0.212 \\
\hline Sex, male vs. female & 0.864 & $0.496-1.505$ & 0.605 & - & - & - \\
\hline CNTN3, high vs. low expression & 0.823 & $0.693-0.978$ & $0.027^{\mathrm{a}}$ & 0.822 & $0.691-0.977$ & $0.026^{\mathrm{a}}$ \\
\hline Treatment, TMZ + radiotherapy vs. radiotherapy & 0.549 & $0.328-0.919$ & $0.023^{\mathrm{a}}$ & 0.534 & $0.310-0.920$ & $0.024^{\mathrm{a}}$ \\
\hline MGMT, methylation vs. no methylation & 3.971 & $2.273-6.937$ & $<0.001$ & 4.324 & $2.401-7.788$ & $<0.001$ \\
\hline
\end{tabular}

${ }^{\text {a }} \mathrm{P}<0.05$. CNTN3, contactin 3; TMZ, temozolomide; MGMT, O6-methylguanine-DNA methyltransferase; HR, hazard ratio; CI, confidence interval.

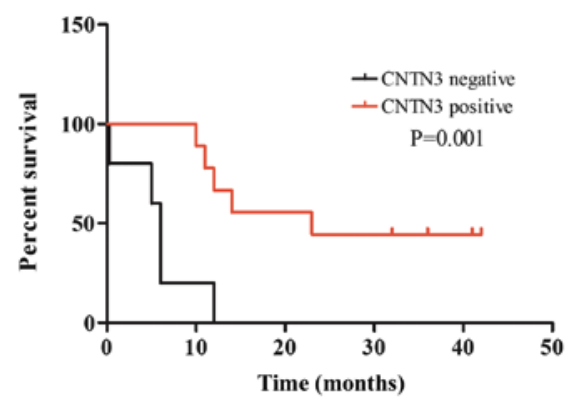

Figure 4. Kaplan-Meier curves of OS. High expression levels of CNTN3 were associated with improved OS. CNTN3, contactin 3; OS, overall survival.

Fig. 3A). Survival analysis was additionally performed on the TCGA-GBM dataset. The median OS time was 12.833 months (95\% CI, 10.897-14.770) in the low expression group and 25.7 months (95\% CI, 17.176-40.950) in the high expression group, with survival being significantly shorter in the low expression group $(\mathrm{P}=0.015$; Fig. 3B).
Table III. Association between CNTN3 and EGFR expression in the tissue microarray.

\section{CNTN3 expression}

EGFR expression Negative, n (\%) Positive, n (\%) P-value

\begin{tabular}{lrrr}
\hline Negative & $28(47.5)$ & $16(76.2)$ & $0.023^{\mathrm{a}}$ \\
Positive & $31(52.5)$ & $5(23.8)$ &
\end{tabular}

${ }^{\mathrm{a}} \mathrm{P}<0.05$. EGFR, epidermal growth factor receptor; CNTN3, contactin 3.

Kaplan-Meier survival analysis was also performed to investigate the prognostic value of CNTN3 protein expression on the survival rate of patients with GBM. The OS rate of patients with tumours that did not express CNTN3 was significantly lower compared with that of patients with tumours positive for CNTN3 expression ( $\mathrm{P}=0.001$; Fig. 4). 
A

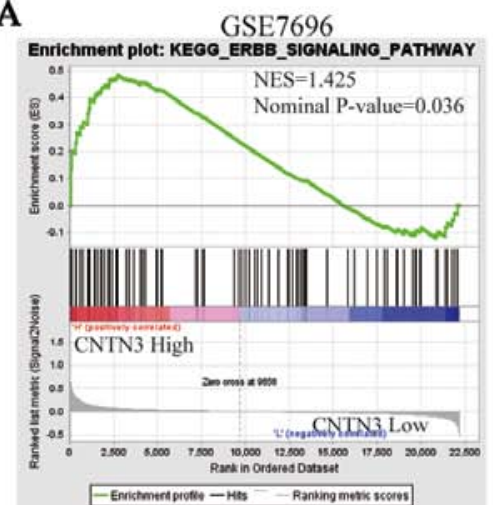

B

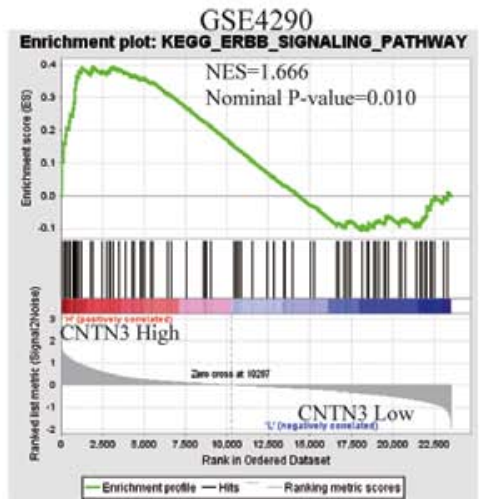

C

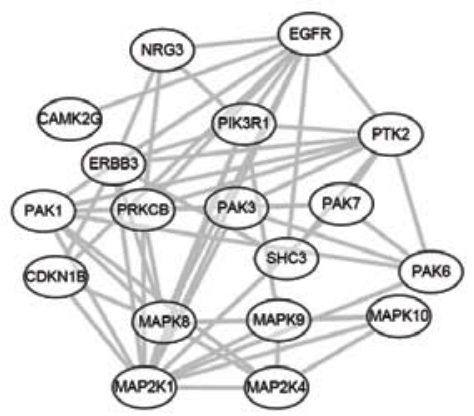

Figure 5. Correlation of CNTN3 expression with the ErbB signalling pathway signatures. Gene sets enrichment analyses showing that $C N T N 3$ expression was associated with ErbB signatures in (A) GSE7696 and (B) GSE4290 datasets. (C) Protein-protein interaction network of proteins involved in the ErbB signalling pathway. CNTN3, contactin 3; ErbB, receptor tyrosine-protein kinase; NES, normalized enrichment score.

A

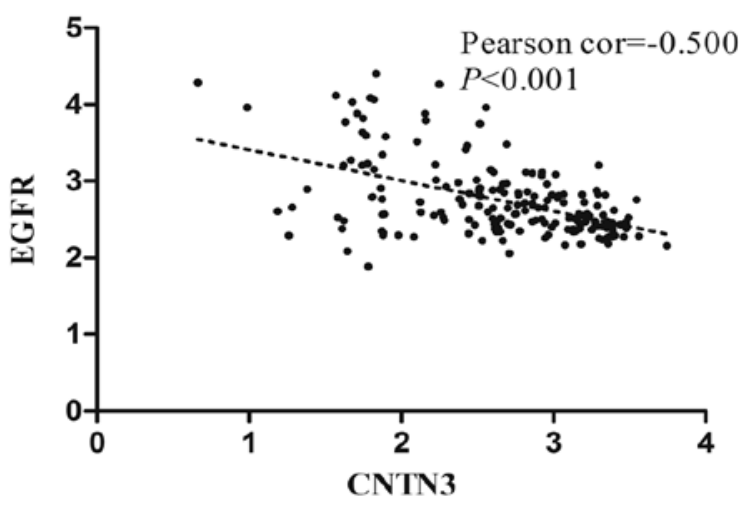

B

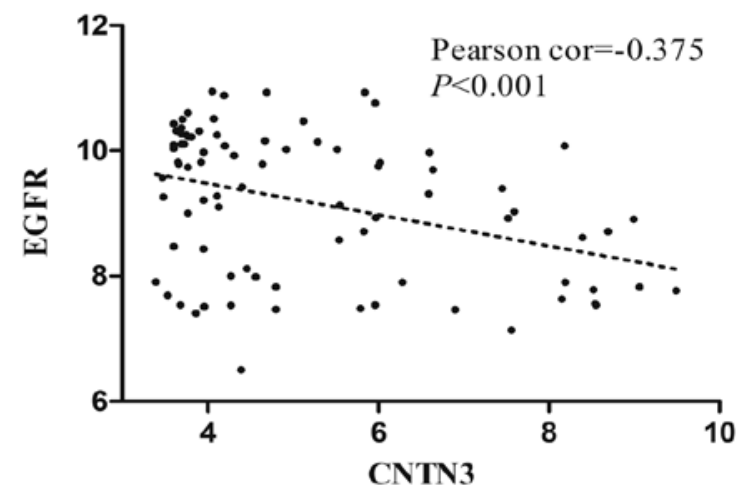

Figure 6. Correlation between $C N T N 3$ and EGFR expression levels. Expression of $C N T N 3$ correlates with EGFR gene expression in (A) GSE4290 and (B) GSE7696 datasets. CNTN3, contactin 3; EGFR, epidermal growth factor receptor; cor, correlation.

Univariate and multivariate Cox regression analyses were performed to confirm the possibility that CNTN3 may be useful as an independent prognostic factor in patients with GBM. Univariate analysis demonstrated that a low expression level of CNTN3 ( $\mathrm{P}=0.027)$, age $\geq 60(\mathrm{P}=0.031)$, treatment with radiotherapy $(\mathrm{P}=0.023)$ and MGMT methylation $(\mathrm{P}<0.001)$ were significantly associated with a poor clinical outcome in GBM cases (Table II). Multivariate analysis further identified that low expression levels of CNTN3 ( $\mathrm{P}=0.026)$, treatment with radiotherapy $(\mathrm{P}=0.024)$ and MGMT methylation $(\mathrm{P}<0.001)$ were independent prognostic factors in patients with GBM (Table II).

Potential molecular mechanisms involving CNTN3 in patients with GBM. To gain insight into the molecular mechanism involving CNTN3 in patients with GBM, GSEA was used to determine whether the CNTN3 gene and associated genes were involved in any oncogenic pathways. The results from the two GEO datasets revealed that $C N T N 3$ was associated with the ErbB signalling pathway (Fig. 5A and B). The 18 genes in this pathway that were associated with $C N T N 3$ were $E G F R$, NRG3, CAMK2G, PIK3R1, PTK2, ERBB3, PAK1, PRKCB, PAK3, PAK7, CDKN1B, SHC3, PAK6, MAPK8, MAPK9, $M A P K 10, M A P 2 K 1$ and $M A P 2 K 4$. The PPI network showed that these genes exhibited complex interactivity with each other (Fig. 5C). EGFR was amplified in $40-50 \%$ of primary GBMs and is thought to participate in tumour cell invasion, angiogenesis and proliferation (21). Therefore, the correlation between the expression of EGFR and CNTN3 was analysed. $E G F R$ expression levels were negatively correlated with CNTN3 expression in the GSE4290 ( $\mathrm{P}<0.001$; Fig. 6A) and GSE7696 ( $\mathrm{P}<0.001$; Fig. 6B) datasets. Additionally, the expression of $C N T N 3$ was positively correlated with the expression of MAPK8, MAPK9 and MAPK10, which are downstream of EGFR (22) (Fig. 7).

EGFR expression in the tissue microarray was also detected. EGFR was primarily located in the cytoplasm and nucleus of neuronal cells (Fig. 8A) and its expression was upregulated in GBM tissues (Fig. 8B). Positive EGFR expression was observed in $15.4 \%$ (2/13) of adjacent normal brain tissues, and in 50.7\% (34/67) of GBM samples $(\mathrm{P}=0.037$, Fig. 8B).

A similar negative correlation was observed between CNTN3 and EGFR expression in the tissue microarray; 52.5\% (31/59) of brain tissue with low CNTN3 expression showed high EGFR expression and $76.2 \%(16 / 21)$ of brain tissue with high CNTN3 expression showed low EGFR expression $(\mathrm{P}=0.045$; Table III). 
A

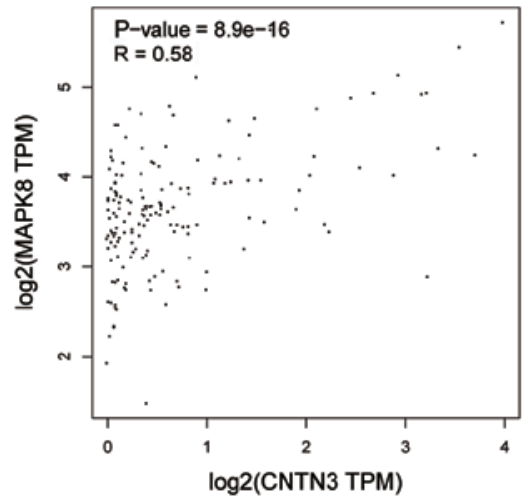

B

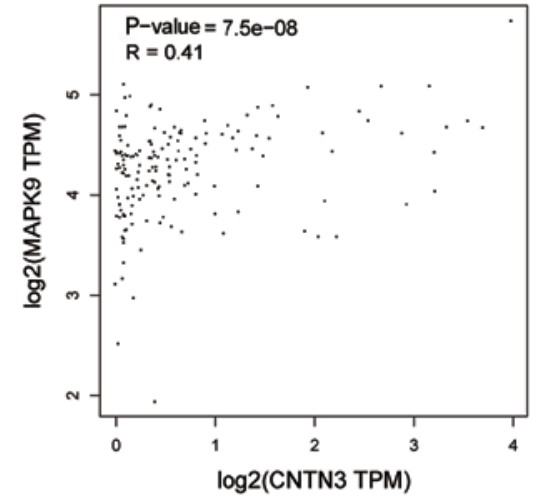

C

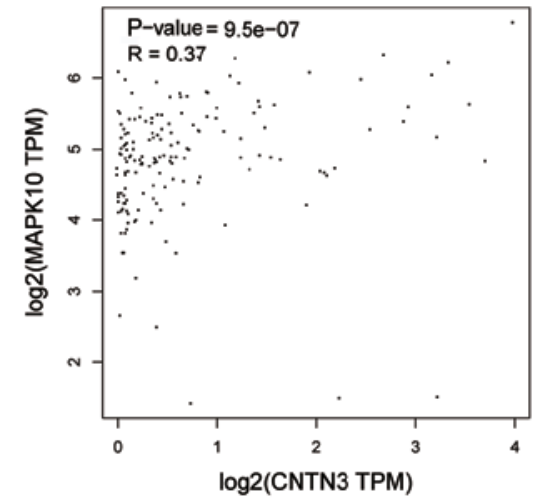

Figure 7. Correlation between $C N T N 3$ expression and (A) MAPK8, (B) MAPK9 and (C) MAPK10 expression. CNTN3, contactin 3; MAPK8, mitogen-activated protein kinase 8; MAPK9, mitogen-activated protein kinase 9; MAPK10, mitogen-activated protein kinase 10; TPM, transcripts per million kilobase.

A

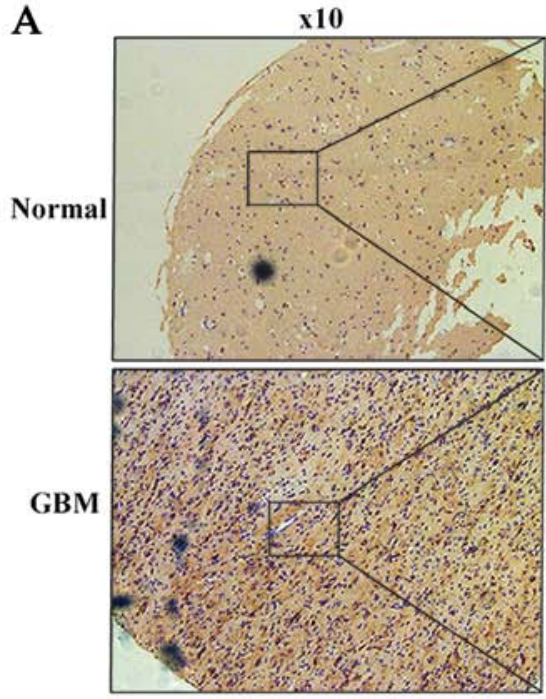

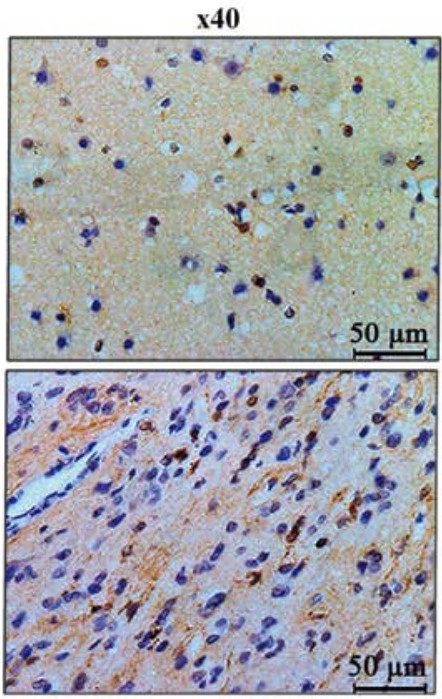

B

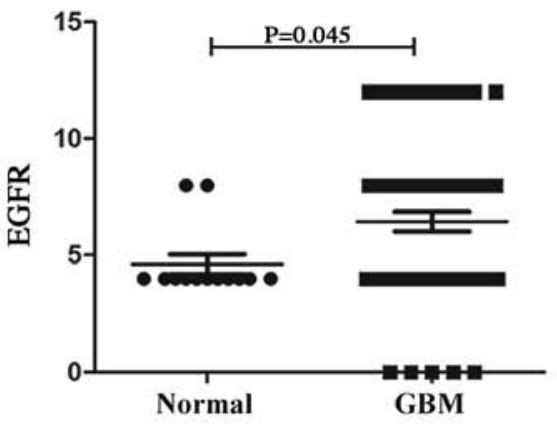

Figure 8. Expression of EGFR in GBM and adjacent normal brain tissues samples. (A) Representative immunohistochemical stains of EGFR expression in GBM and normal brain tissues. (B) EGFR expression was significantly higher in the GBM tissues compared with that in the normal tissues. EGFR, epidermal growth factor; GBM, glioblastoma multiforme.

\section{Discussion}

In the present study, the expression level of the CNTN3 gene was compared in silico between patients with GBM and non-tumour tissues (normal brain tissues and non-tumour tissues from patients with epilepsy) across independent datasets. Expression of the $C N T N 3$ gene was decreased in the GBM samples, and was negatively correlated with OS. Univariate and multivariate analysis revealed that CNTN3 expression was an independent prognostic factor in patients with GBM. These analyses suggest that CNTN3 may serve as a potentially protective factor in GBM. To gain further insights into the role of CNTN3, GSEA analysis was used. CNTN3 was associated with the ErbB signalling pathway. As the most commonly altered gene, EGFR exhibited expression that was negatively correlated with CNTN3. To the best of our knowledge, this is the first study on the clinicopathological and prognostic significance of CNTN3 in GBM.

The function of CNTN3 has not been thoroughly investigated. Cell adhesion/recognition molecules of the immunoglobulin (Ig) superfamily serve vital roles in forming and maintaining the nervous system (8). CNTN3 belongs to the Ig superfamily of proteins and its function is primarily associated with the properties of this family. Akane et al (23), illustrated that the rat ortholog of $C N T N 3$, Cntn 3 , functions in neurite outgrowth-promotion, and when treated with glycidol at $1,000 \mathrm{ppm}$, axonopathy occurred and the expression of CNTN3 was altered. Morales et al (10), found an isolated cryptic proximal interstitial $3 p$ deletion, del(3) (p12.3p13) in a patient with neurodevelopmental delay. Since this deletion site is where the CNTN3 gene is located, it was hypothesized that CNTN3 may serve a role in neuronal development. Subsequently, Hu et al (9) found that deletion or duplication of CNTN6 were associated with neurodevelopmental disorders. However, Monies et al (24) found that possessing additional copies of CNTN3 was not associated with the aforementioned phenotypes. The present study highlights the potential role of CNTN3 in GBM and its use as a prognostic biomarker.

The ErbB receptor family of tyrosine kinases is comprised of four members, EGFR (ErbB1/HER1), ErbB2 (HER2/neu), 
ErbB3 (HER3) and ErbB4 (HER4) (25). Aberrations in EGFR are the most common oncogenic alterations in patients with GBM, and are associated with tumour malignancy and a poor prognosis (26). As EGFR expression was negatively correlated with the expression of $C N T N 3$, CNTN3 may inhibit EGFR expression physiologically. When CNTN3 is downregulated, such as in GBM, EGFR may be aberrantly activated and initiate the ErbB signalling pathway to promote tumour growth and invasion. The signalling pathways mediated by EGFR activation include the RAS/mitogen-activated protein kinase (MAPK)/extracellular signal-regulated kinase, PI3K/protein kinase B, Janus kinase/signal transducer and activator of transcription and protein kinase $\mathrm{C}$ pathways (27). In the present study, the expression of CNTN3 correlated with the expression of MAPK8, MAPK9 and MAPK10, which are downstream of $E G F R$, and therefore these findings suggest that CNTN3 may regulate the properties of GBM by ErbB-MAPK pathway. As the present study was based on results from bioinformatic analyses and tissue microarrays, additional studies are required to determine the association between CNTN3 and the malignant properties of GBM in vitro and in vivo, such as cell proliferation, self-renewal, angiogenesis and drug-resistance.

In conclusion, the present study demonstrates that $C N T N 3$ is downregulated in patients with GBM, and CNTN3 expression is negatively correlated with OS. CNTN3 may be functionally associated with the EGFR signalling pathway, as the expression of EGFR and CNTN3 were negatively correlated. CNTN3 may be used as a prognostic marker and a potential therapeutic target for patients with GBM.

\section{Acknowledgements}

Not applicable.

\section{Funding}

The present study was funded by The Technology Innovation Project of the Chengdu Science and Technology Bureau (grant no. 2018-YF05-00669-SN), The Project of Health and Family Planning Commission of the Sichuan Province (grant no. 18PJ126) and The Project of Health and Family Planning Commission of the Chengdu City (grant no. 2018056).

\section{Availability of data and materials}

The datasets used and/or analyzed during the present study are available from the corresponding author on reasonable request.

\section{Authors' contributions}

YFZ designed the research, analyzed the data, drafted and revised the paper. YBG and LL analyzed the data and revised the manuscript. PY, TTZ, BRP and DFW performed the experiments and re-checked the results. HYZ designed the research. All authors read and approved the final manuscript.

\section{Ethics approval and consent to participate}

Not applicable.

\section{Patient consent for publication}

Not applicable.

\section{Competing interests}

The authors declare that they have no competing interests.

\section{References}

1. Shergalis A, Bankhead A III, Luesakul U, Muangsin N and Neamati N: Current challenges and opportunities in treating glioblastoma. Pharmacol Rev 70: 412-445, 2018.

2. Lefranc F, Le Rhun E, Kiss R and Weller M: Glioblastoma quo vadis: Will migration and invasiveness reemerge as therapeutic targets? Cancer Treat Rev 68: 145-154, 2018.

3. Gallego O: Nonsurgical treatment of recurrent glioblastoma. Curr Oncol 22: e273-e281, 2015.

4. Shimoda Y and Watanabe K: Contactins: Emerging key roles in the development and function of the nervous system. Cell Adh Migr 3: 64-70, 2009.

5. Yoshihara Y, Kawasaki M, Tani A, Tamada A, Nagata S, Kagamiyama $\mathrm{H}$ and Mori K: BIG-1: A new TAG-1/F3-related member of the immunoglobulin superfamily with neurite outgrowth-promoting activity. Neuron 13: 415-426, 1994.

6. Connelly MA, Grady RC, Mushinski JF and Marcu KB: PANG, a gene encoding a neuronal glycoprotein, is ectopically activated by intracisternal A-type particle long terminal repeats in murine plasmacytomas. Proc Natl Acad Sci USA 91: 1337-1341, 1994.

7. Luo C, Li Y, Zhang X, Zhang Y, Zhang H, Chen C, Xu Z, Cui P, $\mathrm{Hu} \mathrm{S}$, Yang $\mathrm{H}$ and Dong W: DNA sequence comparative analysis of the 3pter-p26 region of human genome. Sci China C Life Sci 48: 34-40, 2005.

8. Mohebiany AN, Harroch S and Bouyain S: New insights into the roles of the contactin cell adhesion molecules in neural development. Adv Neurobiol 8: 165-194, 2014.

9. Hu J, Liao J, Sathanoori M, Kochmar S, Sebastian J, Yatsenko SA and Surti U: CNTN6 copy number variations in 14 patients: A possible candidate gene for neurodevelopmental and neuropsychiatric disorders. J Neurodev Disord 7: 26, 2015.

10. Morales C, Mademont-Soler I, Armengol L, Milà M, Badenas C, Andrés S, Soler A and Sánchez A: Characterization of a 5.8-Mb interstitial deletion of chromosome $3 p$ in a girl with $46, X X, \operatorname{inv}(7)$ dn karyotype and phenotypic abnormalities. Cytogenet Genome Res 125: 334-340, 2009.

11. Yoshihara Y, Kawasaki M, Tamada A, Nagata S, Kagamiyama $\mathrm{H}$ and Mori K: Overlapping and differential expression of BIG-2, BIG-1, TAG-1, and F3: Four members of an axon-associated cell adhesion molecule subgroup of the immunoglobulin superfamily. J Neurobiol 28: 51-69, 1995.

12. Bouyain $S$ and Watkins DJ: The protein tyrosine phosphatases PTPRZ and PTPRG bind to distinct members of the contactin family of neural recognition molecules. Proc Natl Acad Sci USA 107: 2443-2448, 2010.

13. Wu Q, Wang C, Guo L, Ge Q and Lu Z: Identification and characterization of novel microRNA candidates from deep sequencing. Clin Chim Acta 415: 239-244, 2013.

14. Sun L, Hui AM, Su Q, Vortmeyer A, Kotliarov Y, Pastorino S, Passaniti A, Menon J, Walling J, Bailey R, et al: Neuronal and glioma-derived stem cell factor induces angiogenesis within the brain. Cancer Cell 9: 287-300, 2006.

15. Murat A, Migliavacca E, Gorlia T, Lambiv WL, Shay T, Hamou MF, de Tribolet N, Regli L, Wick W, Kouwenhoven MC, et al: Stem cell-related 'self-renewal' signature and high epidermal growth factor receptor expression associated with resistance to concomitant chemoradiotherapy in glioblastoma. J Clin Oncol 26: 3015-3024, 2008.

16. Xia L, Su X, Shen J, Meng Q, Yan J, Zhang C, Chen Y, Wang H and Xu M: ANLN functions as a key candidate gene in cervical cancer as determined by integrated bioinformatic analysis. Cancer Manag Res 10: 663-670, 2018.

17. Team RC: R: A language and environment for statistical computing. $R$ Foundation for Statistical Computing, Vienna, Austria. ISBN 3-900051-07-0, URL http://www.R-project.org/, 2012.

18. Yu J, Wu X, Huang K, Zhu M, Zhang X, Zhang Y, Chen S, Xu X and Zhang Q: Bioinformatics identification of lncRNA biomarkers associated with the progression of esophageal squamous cell carcinoma. Mol Med Rep 19: 5309-5320, 2019. 
19. Subramanian A, Tamayo P, Mootha VK, Mukherjee S, Ebert BL, Gillette MA, Paulovich A,Pomeroy SL, Golub TR, Lander ES and Mesirov JP: Gene set enrichment analysis: A knowledge-based approach for interpreting genome-wide expression profiles. Proc Natl Acad Sci USA 102: 15545-15550, 2005.

20. Shannon P, Markiel A, Ozier O, Baliga NS, Wang JT, Ramage D, Amin N, Schwikowski B and Ideker T: Cytoscape: A software environment for integrated models of biomolecular interaction networks. Genome Res 13: 2498-2504, 2003.

21. Talasila KM, Soentgerath A, Euskirchen P, Rosland GV, Wang J, Huszthy PC, Prestegarden L, Skaftnesmo KO, Sakariassen PØ, Eskilsson E, et al: EGFR wild-type amplification and activation promote invasion and development of glioblastoma independent of angiogenesis. Acta Neuropathol 125: 683-698, 2013.

22. Wee P and Wang Z: Epidermal growth factor receptor cell proliferation signaling pathways. Cancers (Basel) 9: pii: E52, 2017.

23. Akane H, Saito F, Shiraki A, Imatanaka N, A kahori Y, Itahashi M, Wang L and Shibutani M: Gene expression profile of brain regions reflecting aberrations in nervous system development targeting the process of neurite extension of rat offspring exposed developmentally to glycidol. J Appl Toxicol 34: 1389-1399, 2014.
24. Monies D, Abouelhoda M, AlSayed M, Alhassnan Z, Alotaibi M, Kayyali H, Al-Owain M, Shah A, Rahbeeni Z, Al-Muhaizea MA, et al: The landscape of genetic diseases in Saudi Arabia based on the first 1000 diagnostic panels and exomes. Hum Genet 136: 921-939, 2017.

25. Berezowska S and Schlegel J: Targeting ErbB receptors in high-grade glioma. Curr Pharm Des 17: 2468-2487, 2011.

26. Wang L, Liu Q, Zhao H, Cui K, Yao L, Nie F, Jin G, Hao A and Wong ST: Differential effects of low- and high-dose GW2974, a dual epidermal growth factor receptor and HER2 kinase inhibitor, on glioblastoma multiforme invasion. J Neurosci Res 91: 128-137, 2013.

27. An Z, Aksoy O, Zheng T, Fan QW and Weiss WA: Epidermal growth factor receptor and EGFRvIII in glioblastoma: Signaling pathways and targeted therapies. Oncogene 37: 1561-1575, 2018.

This work is licensed under a Creative Commons

Attribution-NonCommercial-NoDerivatives 4.0

International (CC BY-NC-ND 4.0) License. 